\title{
DISSEMINATION OF MHV4 (STRAIN JHM) INFECTION DOES NOT REQUIRE SPECIFIC CORONAVIRUS RECEPTORS
}

\author{
T. M. Gallagher(1), M. J. Buchmeier(1) and \\ S. Perlman(2) \\ (1)Division of Virology \\ Department of Neuropharmacology \\ The Scripps Research Institute \\ La Jolla, CA 92037 \\ (2)Departments of Pediatrics and Microbiology \\ University of Iowa \\ Iowa City, IA 52242
}

\begin{abstract}
In this report, we demonstrate the syncytial spread of MHV4 (strain JHM) infection through non-murine cell cultures which lack a specific MHV4 receptor and are therefore resistant to infection by free virions. This was achieved by allowing infected murine cells to settle onto confluent monolayers of non-murine cells in a straightforward infectious center assay. Receptor-independent syncytium formation induced by cells expressing the MHV4 spike (S) from recombinant vaccinia viruses (VV) indicated that spread was mediated by this coronavirus glycoprotein. We conclude that the S protein of MHV4 is so potently fusogenic that it does not require prior binding to a virus-specific surface receptor to induce fusion of closely-opposed plasma membranes.
\end{abstract}

\section{INTRODUCTION}

It is well known that the murine coronaviruses exhibit tropism for murine hosts; these viruses are transmissible to rats or monkeys (1) only under highly artificial conditions of intracerebral inoculation. In addition, considerable evidence indicates variation in the susceptibility of murine cell types to murine coronavirus infection (2). Tropism for murine cells appears to be due to the absence of a suitable plasma membrane receptor for murine coronavirus on non-murine cells (3). The additional restriction to selected mouse cell lines 
may be due to requirements subsequent to receptor binding, such as those necessary for the appropriate exposure of infectious RNA to the cytosol $(4,5)$. Such species and tissue preference contrasts with the extended host range of viruses from other families. For example, the myxoviruses bind and productively infect a wide range of cell types, presumably because many configurations of ubiquitous sialic acid are acceptable receptor ligands (6), and because subsequent genome delivery is relatively unrestricted.

However, two observations suggested to us that murine coronavirus infections may actually involve more cell types than is commonly believed. First, for years now we have observed clear evidence of syncytia formation in non-murine cells infected with VV-S recombinants, suggesting that specific spike:receptor binding is not a prerequisite for MHV-induced fusion activity. This finding has in fact been recently documented for insect and rabbit cells $(7,8)$. Second, Murray et al. (9) recently demonstrated MHV infection of primates, with resultant clinical symptoms, upon intracerebral inoculation with lysates of infected murine cells. This result, along with a similar finding in rats (10) suggested an unexpectedly large in vivo host range for MHV. These discoveries prompted our investigation into whether an authentic MHV4 infection could disseminate and amplify in cultured cell monolayers of non-murine origin.

\section{METHODS AND RESULTS}

\section{Expression of MHV4 spike from recombinant VV-infected cells results in syncytial spread on non-murine cells}

Previous studies (11) involved synthesis of the MHV4 S glycoprotein from cDNA inserted in a plasmid containing a bacteriophage T7 RNA polymerase promoter. The plasmid, designated pGEM4Z-S, successfully expressed $\mathrm{S}$ molecules when transfected into cells infected with vTF7.3 (12) a recombinant VV that provided requisite T7 RNA polymerase activity. S protein expressed in this manner clearly induced syncytium formation on DBT (mouse) cell monolayers, but failed to fuse Hela (human), BHK (hamster) or RK13 (rabbit) cell monolayers, suggesting a requirement for MHV receptor in fusion. These findings were in agreement with previous reports involving expression of murine coronavirus S glycoproteins from weak VV promoters (13), and most likely reflected poor translational activity of the RNA transcribed from the pGEM T7 promoter (14).

In an attempt to increase the level of glycoprotein expression from cDNA, we excised $\mathrm{S}$ cDNA from pGEM4Z-S and inserted it into two different vaccinia virus (VV) insertionexpression vectors, pTM1 (14) and p1200 (15). pTM1 carries both a T7 RNA polymerase promoter and an efficient picornaviral ribosome binding site, thereby providing the cis elements necessary for high efficiency, early transcription and translation of insert cDNA. p1200 harbors a late cowpox promoter whose activation in VV-infected cells gives rise to abundant late transcripts.

For insertion into pTM1, PCR mutagenesis (16) was performed to create an NcoI site at the $\mathrm{S}$ initiation codon, and then the S cDNA (Ncol-BamHI) was ligated into the polylinker region. Insertion into p1200 involved modification of S cDNA (HindIIIBamHI) using linkers to generate ends compatible with ClaI overhangs followed by ligation into the unique ClaI site of the vector. Standard methods involving homologous recombination and bromodeoxyuridine selection (17) were used to prepare VV-spike (vTM1-S and v1200-S) recombinants. 


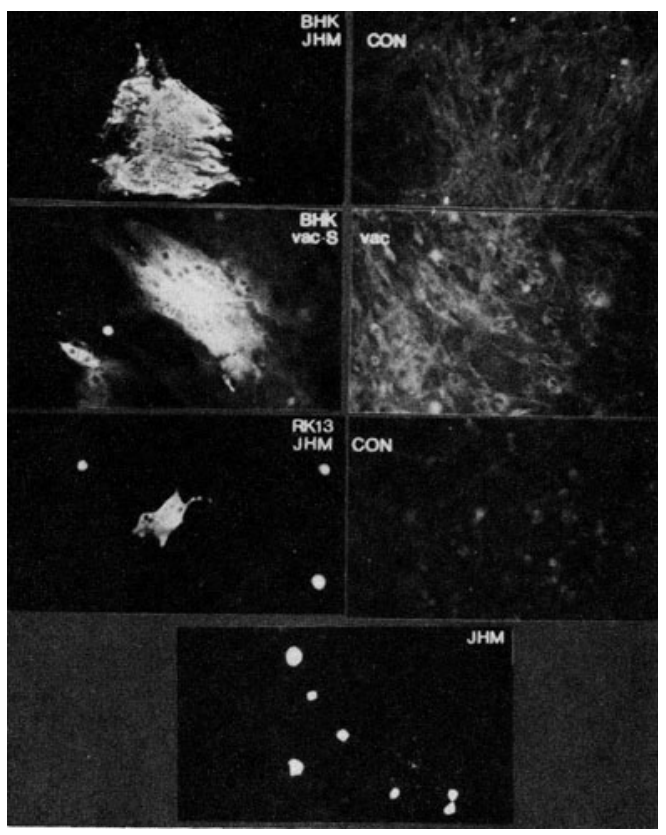

Figure-1. MHV can spread to resistant BHK and RK13 cells. After trypsinization, DBT cells were resuspended to $10^{6}$ cells/ml and either mock-infected ("con") or infected with either MHV (0.5 PFU/cell) ("JHM") or vTF7.3 (which expresses T7 RNA polymerase) and vTM1-S ("vac-S") or vTF7.3 alone ("vac"). After $1 \mathrm{hr}$ at $37^{\circ} \mathrm{C}$, cells were extensively washed to remove unbound virus. $1.0 \times 10^{5}$ cells aliquots were then seeded onto confluent lawns of either BHK rows (1, 2), RK13 (row 3) or onto empty wells (row 4). After $11 \mathrm{hrs}$ (cells dually infected with VV recombinants) or $22 \mathrm{hrs}$ (cells infected with MHV), cells were fixed with $10 \%$ formalin, permeabilized with $2 \%$ NP40 and incubated with a mixture of murine monoclonal antibodies specific for the nucleocapsid, matrix and spike proteins. Antiviral antibody was detected with fluoroscein-conjugated goat anti-mouse antibody $\left(\mathrm{Fab}^{\prime}\right)^{2}$ fragment. 
Coomassie-stained electropherograms representing the protein from cells dually infected with vTF7.3 and vTM1-S did not reveal S glycoprotein (data not shown), indicating that $\mathrm{S}$ expression was substantially lower than previously reported for this system (14). Despite this finding, we did observe fusion of Hela, BHK and RK13 cells by 12 hours postinfection (hpi). Figure 1 depicts syncytium formation on a BHK cell monolayer. Similar results (not shown) were obtained from v1200-S infected cells at 48 hpi. Thus, it appeared that the requirement for a specific plasma membrane ligand for $S$ in the fusion process could be overcome by increasing the surface density of the $\mathrm{S}$ fusogen.

\section{Expression of MHV4 spike from authentic virus-infected cellsresults in syncytial spread on non-murine cells}

Discovery of vS-mediated syncytium formation across a broad spectrum of cell types led to the obvious question of whether S expressed from authentic MHV4 could function similarly. Human, hamster and rabbit cells are known to be resistant to infection with MHV virions, and using hamster cells, this resistance has been shown to be due to lack of cell surface receptor (3). To test the fusion function of S expressed from MHV4 we performed infectious center assays. Susceptible murine DBT cells were infected in suspension with MHV4 and were seeded onto confluent monolayers of DBT, BHK or RK13 cells. Foci of fused cells containing viral antigens developed in all three different cell monolayers. The results with BHK and RK13 cells are shown in Figure 1. By quantitating immunofluorescence signals, we found that essentially all productively infected DBT cells were able to form foci on all cell lines. Syncytium size, however, varied with cell type, with DBT being largest (50-100 nuclei/focus by $11 \mathrm{hr}$ pi), BHK being intermediate (20-30 nuclei/focus by $22 \mathrm{hr}$ pi) and RK13 being smallest (1-10 nuclei/focus by $22 \mathrm{hr} \mathrm{pi)}$.

Progeny virus production in these spreading foci was assayed at $22 \mathrm{hpi}$ (Table 1). On average, the respective yields from DBT, BHK and RK13 cells were 7800, 250 and 21 times higher than from control cultures of infected cells plated in the absence of an underlying cell monolayer.

Table 1. Progeny virus titers after recruitment of cell monolayers into MHV4 infection.

$\begin{array}{cc}\text { Underlying cell monolayer } & \text { Titer(a) } \\ \text { None (Control) } & 2.47+/-0.31 \\ \text { RK13 } & 3.79+/-0.28 \\ \text { BHK } & 4.87+/-0.56 \\ \text { DBT } & 6.36+/-0.27\end{array}$

$10^{5}$ DBT cells infected in suspension with MHV4 $(0.5 \mathrm{FU} / \mathrm{cell})$ were diluted at $1 \mathrm{hr} \mathrm{pi}$ into $3 \mathrm{ml}$ of complete growth medium and overlaid on to $10 \mathrm{~cm}^{2}$ plates containing the indicated confluent underlying monolayers. After $22 \mathrm{hrs}$, virus was released from cells by three freeze-thaw cycles and the resulting extracts were titered on DBT indicator cells.

(a) Titers are expressed as geometric means $+/$ - one standard deviation per ml. 


\section{DISCUSSION}

\section{Receptor-independent dissemination of infection may have in vivo relevance}

Our in vitro results suggest that MHV4 may spread within infected animals without requirement for receptor. Infection of this type may operate in the rat, which lacks MHV receptor (18), yet can by infected if cell lysates or brain tissue homogenates are used as inocula (10). Our results also suggest an important role for multinucleated giant cells in the infected animal. Limited data concerning this possibility is available (19), and careful studies employing mutants which vary in fusogenicity, as well as in situ hybridization and immunohistochemistry techniques, will be required to identify in vivo spread of infection.

\section{Virions and infected cells may establish infection in Fundamentally different ways}

Considerable concentration of virus particles from supernatant fluids to cell surface is a requisite initial step in infection by virions. In contrast infected cells display exocytosed $S$ glycoprotein at the plasma membrane at high density. Only low affinity interactions permitting juxtaposition of opposing cells would be required to bring $S$ into range for fusion activity. Adhesion molecules normally present on cell surfaces may promote the obligate interactions.

Receptor-independent spread only requires the presenceof a fusogenic surface molecule and could potentially be important in the spread of paramyoxoviruses, herpes viruses and lentiviruses. It has been shown recently that the fusogenic gp41 transmembrane protein of HIV is capable, in the absence of all other HIV proteins, of initiating syncytium formation in normally resistant cells (20). This suggests that receptor-independent spread may be functional in other systems under the appropriate circumstances.

The mechanism of murine cell resistance to infection by MHV4 virions remains unknown, although previous results have pointed toward the importance of events after receptor binding but before RNA synthesis $(2,4,5)$. Such events include virion:cell membrane fusion, internalization of virus and nucleocapsid delivery to the cytoplasm. Thus it remains to be determined whether the delivery of infected cytosol to resistant cells can be achieved by cell:cell fusion.

\section{Functional domains of the spike can be defined}

With the infectious center assay described in this report it will be possible to study the membrane fusion function of individual spike glycoproteins in the absence of their receptor binding function. Previous crude assays comparing the syncytium-forming capacity of variant $S$ proteins (11) did not account for variations between the proteins for their cognate receptor. Variant spikes on the surface of vS-infected cells can now be tested in the absence of receptor. These assays will permit unambiguous identification of those residues participating in the membrane fusion process.

Acknowledgements: The authors thank Chris Baugh and Michelle Zandonatti for technical assistance. We also thank Dr. B. Moss for providing vTF7.3 and pTM1 and Dr. D.J. Pickup for providing p1200. This work was performed while S.P. was on sabbatical 
leave at The Scripps Research Institute. Supported by grants from the NIH--NS 12428, NS 22347 (MJB) and NS 24401 (SP), and from the Multiple Sclerosis Society (SP). SP was supported by a Research Career Development Award from the NIH. This is publication No. 8583-NP from the Scripps Research Institute.

\section{REFERENCES}

1. H. Wege, S. Siddell, and V. ter Meulen. Current Topics in Microbiology and Immunology 99:165-200 (1982).

2. L. Sturman, and K. Holmes. Adv. in Virus Res. 28: 35-112 (1985).

3. G. Dveksler, C. Pensiero, C. Cardellichio, R. Williams, G. Jiang, K. Holmes, and C. Dieffenbach. J. Virol. 65:6881-6891 (1991).

4. I. Shif, and F. Bang. J. Exp. Med. 131: 843-850 (1970).

5. C. Kooi, L. Mizzen, C. Alderson, M. Daya, and R. Anderson. J. Gen. Virol. 69: 1125-1135 (1988).

6. J. Paulson, J. Sadler, and R. Hill. J. Biol. Chem. 254:2120-2124 (1979).

7. F. Taguchi, T. Ikeda, and H. Shida. J. Gen. Virol. 73:1065-1072 (1992).

8. D. Yoo, M. Parker, L.A. Babiuk. Virology 180:395-399 (1991).

9. R. Murray, G. Cai, K. Hoel, J. Zhang, K. Soike, G. Cabirac. Virology. 188: 274-284 (1992).

10. O. Sorensen, D. Perry, and S. Dales. Arch. Neurol. 37:478-484 (1980).

11. T. Gallagher, C. Escarmis, and M. Buchmeier. J. Virol. 65:1916-1928 (1991).

12. T. Fuerst, E. Niles, F. Studier, and B. Moss. Proc. Natl. Acad. Sci. USA 83: 81228126 (1986).

13. H. Vennema, L. Heijnen, A. Zijderveld, M. Horzinek, and W. Spaan. J. Virol. 64: 339-346 (1990).

14. O. Elroy-Stein, and B. Moss. Proc. Natl. Acad. Sci. USA 87: 6743-6747 (1990).

15. D. Patel, C. Ray, R. Drucker, and D. Pickup. Proc. Natl. Acad. Sci. USA 85: 94319435 (1988).

16. H. Kadowaki, T. Kadowaki, F. Wondisford, and S. Taylor. Gene 76: 161-166 (1989).

17. M. Mackett, G. Smith, and B. Moss. J. Virol. 49:857-864 (1984).

18. K. Holmes, R. WIlliams, C. Cardellichio, S. Comptorn, C. Stephensen, S. Shyder, M. Frana, G-S. Jiang, A. Smith, R. Knobler. Adv. Exp. Med. Biol. 276: 37-44 (1990).

19. N. Goto, K. Takahashi, K. Huang, K. Katami, and K. Fujiara. Japan. J. Exp. Med. 49: 169-177 (1979).

20. L.G. Perez, M.A. O'Donnell, E.B. Stephens. J. Virol. 66:4134-4143 (1992). 